\title{
Interview Method and Thought on the Intangible Cultural Heritage Inheritors of Jiangxi Traditional Music
}

\author{
Jiaqi Mei \\ Jiujiang College \\ Jiujiang, China
}

\begin{abstract}
Interview is not only the most common form of interpersonal interaction in social life, but also the most basic way of data collection in inheritors' oral history research of intangible cultural heritage, which is also a process of knowledge construction in its social essence. It is communication and interaction between the oral and non-oral information on the past historical events by the general public.
\end{abstract}

Keywords-Jiangxi traditional music; inheritors of intangible cultural heritage; interview

\section{INTRODUCTION}

Inheritors are the soul of the intangible cultural heritage project. To protect the inheritors of the intangible cultural heritage of Jiangxi traditional music is equivalent to protect the soul of the intangible cultural heritage, which is irreplaceable by other protection methods. Thus, it is an important work to record the activities of the inheritors and keep the shadow of the them.

The oral history of the inheritors on the intangible cultural heritage is a kind of method to research the history by collecting and using the oral historical documents. Interview is the basic way for collecting the oral historical documents, and also the basic premise of the research on oral history. It can be said that if there is no interview, the research on the oral history of the inheritors about the Jiangxi traditional music intangible cultural heritage will be the wood without root and water without source.

Today, the use of interviews to gain access to information is becoming more and more common, so that society being lived by the researchers can also be called as "interview society." Thus, the oral researchers should be increasingly aware that oral interviews are not neutral data collection tools, but the negotiated results according to the specific context of the discourse by two or more individuals. The interview to the inheritors of the intangible cultural heritages of traditional music in Jiangxi province not only includes the traditional daily life activities, but also the routine of people's daily life. This shows that the interview actually has been deeply involved in the daily life, and the construction of the interpersonal communication rules.

Interviews to the inheritors of the traditional music

Fund project: Jiangxi Province Arts and Sciences Planning Project "Research on the oral history of the inheritors for the music intangible cultural heritage of Jiangxi Province" No.: YG2015175, this article is one of the research results. intangible heritage have become a way of contemporary narrative. In the interview, people tell life history to the investigators. Interviews, as a way for collecting data, have become a "universal way of systematic investigation", and it seems that all types of problems - private, sensitive, exploratory, distressing, and blaming - can be as the target of an interview, and are permitted in the context of the interview. Almost all interviews, from description, inquiry, help, examination to evaluation, can be sought in various forms described through interviews, regardless of their purpose.

Interview is not only the most common form of interpersonal interaction in social life, but also the most basic way of data collection in inheritors' oral history research of intangible cultural heritage, which is also a process of knowledge construction in its social essence. It is communication and interaction between the oral and non-oral information on the past historical events by the general public. So that individuals in society, disadvantaged groups have the opportunity to interpret their own life experience by their own language and perspective.

Interviews can be either casual or formal; can be in form of face-to-face or non face-to-face, such as telephone interviews or online interviews. The interview can be the dialogue between two individuals or more ones, so the types of interview are quite various, you can use different forms for oral interviews. That is to say, the oral interview method is not single, but various, the interview methods can be chosen according to the oral history theme.

\section{STRUCTURAL INTERVIEW}

The structural interview is also called as "standardized interview" or "formal interview." Structural interview means that the researchers use a set of predefined structural questions during the interview process to collect the document.

The so-called "structure" belonging to structural interview refers to the questions needed to be answered have formed a certain logical index system, interview outline is the embodiment of this system, the questions needed to be solved during the interview mainly about gaining the detail information and document related with these indicators; on the contrary, the unstructured questions refers to the logical structure of target questions needed to be researched is not 
clear or clear. The target questions needed to be solved during the interview process are to solve the solve logical structure of the target questions and the specific information of the key questions that constitutes the logical structure. In general, researchers usually deal with structural problems through unstructured interviews, and then solve the inherent problems of constituent elements through structural interviews.

In a structural interview, the interviewer basically asks all the interviewees about the same, pre-designed questions. In addition to the few open-ended questions, the answers to the questions asked by the researchers are often very limited, and there will be no major changes in the answers. Structural interviews are mainly based on the stimulus of interviewees receiving similar question situations during the interview, so that the data collected by the researcher will not be too deviated from the subject. This means that structural interview is to pre-design the questions for investigators to understand the thoughts, opinions, and attitudes of the interviewees, and to mitigate possible biases by using pre-structured questions and interview normalization procedures.

In structural interviews, most questions and answers are inflexible. Interviewers design the interviewing content as questionnaires according to the pre-designed coding scheme, recorded answer, or the requirements of the questions to control the interview method and progress in form of the questionnaires, take the questionnaire as play to address the interview structurally, normally and gradually. Therefore, the interviewer should take all the interviewed questions and interviewees as same, and ask the same question in the same order, it is forbidden to adjust or modify the content during the interview process to affect the accuracy of the questions.

The researchers can construct the ideal structured interview step by step via considering the following elements.

First, pay attention to the behaviors and actions of the interviewees during the interview process, try to reduce the possible influence of the social hope to the interviewee during the interview process.

Second, fix the research and interview type according to the researched questions and research purpose, whether a faceto-face interview or a telephone interview to collect data is appropriate; meanwhile, arrange according to the interview content and order. It is necessary to avoid asking reading skills, or human factors to influence the intended outcome of the interview in the interview process.

\section{UNSTRUCTURED INTER VIEW}

Unstructured interviews, also known as non-structural interviews, non-standard interviews, informal interviews, or open-ended interviews.

The unstructured interview means that the researcher does not use the fixed question as the guide for the interview during the interview, and does not need to design a standardized interview outline in advance as the guiding guide for the interview.

The main purpose of the structural interview is to emphasize how the researcher collects the right and correct information. However, the unstructured interview focuses on how to understand the complex life phenomenon or the social behavior, the daily life and the historical experience in the natural context of the story. Therefore, each question can be flexibly used and adjusted according to the characteristics of the interviewee or the interviewer's situation. During the interview, the interviewer should try to avoid influencing the interviewee by guiding or hinting. The interviewee should try his best to express his views and opinions in the natural relaxed context.

There are three types of non-structured interviewing techniques:

1. Cycle mode: The interviewing techniques of the cycle mode is a form of questioning, which is more like a general pattern of conversation, in which people and situations are treated as unique. So the direction of the conversation will be adjusted according to the interaction of each interview.

2. Hopper-type: The process of questioning begins with a general or broader approach by involving the respondents in conversations and then slowly closing to core or specific issues This is a gradual focus of the interview; the interviewers gradually shifted the focus of the questions to the main purpose and target questions via the conversation between them.

3. Tell story type: The first type of inquiry in this interview is a descriptive question, asking for some description of the activity, characteristic, or sensation. The second type of question is to ask the interviewee to do more complicated analysis on the raw data of their experience before answering questions, such as asking questions needed to be summarized, categorized, abstracted, quantified, or interpreted.

\section{SEMI-STRUCTURED INTER VIEW}

Semi-structured interview is a way of collecting document between the structural and unstructured interview. Before interviewing, the researcher should design the outline of the interview according to the question and purpose of the study, as the guideline of the interview.

However, during the entire interview, the interviewer does not have to follow the interview program to do interview. Usually, the interviewer can also make some corresponding adjustment according to the actual situation, the inquiry procedure, the questioning method and even the specific content of the question.

\section{FIELD INTERVIEW STYLE}

Here, the "field interview style" means that the interview method which is unexpectedly produced during the anthropological field work for adapting to the needs of it. Compared with other types of interview methods, the time and place of other interview methods are deliberately arranged, while the field interview rules generally do not need clear interview space and time. 


\section{THE Focus(GROUP) INTERVIEW}

This interview refers to the interview that one or two researchers interview a group of people at the same time, and discuss the focusing questions by the interaction among the group members. Focus group interviews make the interview itself become as the object of study, do a collective discussion to the researched issues, and build knowledge in the interview process in a collective way.

The methods of group interview in sociology and the method of oral history in history provide a prototype for group interview. This method is widely used in the press, sociology, etc., some market research is also widely used this method.

This method of interview can quickly activate some predictions or unanticipated ideas by the discussion of group interaction. These advantages, however, are precisely the shortcomings of focus group interviews, which focus only on groups of high homogeneity or social groups, so that oral interviews and related issues can be discussed to get the desired interview results in a shorter time.

On the contrary, individual oral interviews are timeconsuming, but because of the heterogeneous experience of interviewees, it is very easy for interviewers to grasp the differences in the causes of memory, life, life history, etc. , thus the collected oral information is more detailed and rich than the content gotten from the focus group interview method.

\section{CONCLUSION}

In short, all kinds of interviews, if successful, they are basically able to reflect the following seven functions: the establishment of interview style; the establishment of interactive relationships; evoke personal memories; establish a bridge of communication; grant the discourse authority to the respondents; provide contextual information for analyzing the problem; and weave a conversation thread for thematic research questions. After the type of interview and the type of questions are basically defined, the researcher will need to make one or more preliminary interviews or testing interviews. Once the interviewee is selected and the outline of the interview is designed, the researcher must try to understand the situation of the interview and the specific situation of the interviewee, and try to figure out the interview role and practice the interview. During the interview, the researcher must make a short field summary record. These records must include: observation of the interviewee, observation of the surroundings, observation of the interview content and process. When the formal interview ends and the researcher turns off the recording device, it may be desirable to maintain follow-up interview for about five minutes of. At this time, researchers can be alert to the emergence of new counseling, and also gives a friendly feeling of the end.

\section{REFERENCES}

[1] Ping Wan Oral History of the Old Artist[M]. People 's Publishing House. 2014

[2] Xiangping Li, Yangbo Wei. Research Method of Oral History[M]. Shanghai People's Publishing House. 2010
[3] Yizhuang Ding, Run Wang. Reading for Oral History[M]. Beijing University Press. 2011

[4] Li Yuan, Jun Gu. Oral History of the Inheritors on Non-material Cultural Heritage of Beijing[M]. Capital Normal University Press. 2015

[5] Huawen Chen. Keep the Inheritors[M]. Zhejiang Industrial and Commercial University Press. 2013

[6] Mingjun Liao, Minkang Yang. Protection on Traditional Music and Non-material Culture Heritage[J]. National Art. 2008(01)

[7] Hong Dai. The Inheritors of the Traditional Music in the eyes of Nonmaterial Culture Heritage Protection [J]. Xinghai Music College Journal 2008(01)

[8] Guangyu Feng. The Protection of the Non-material Culture Heritage and the Heritage of the Chinese Traditional Music[J]. Shenyang Music College Journal, 2006(02)

[9] Gang Pan. The Principle and Method of the Inheritors' Oral History about the Non-material Culture Heritage under the Folk Custom Context[J]. Yunnan Normal University Journal. 2015(03)

[10] Xinyi Liu. Data Base Constructing Current Status and the Image of the Inheritors Oral History on Non-material Cultural Heritage in Guizhou Province[J] Guizhou Normal University. 2013(08) 